\title{
Message from the special issue editors
}

\author{
Luiz Eduardo Buzato • Elias P. Duarte Jr. • \\ Mohamed Kaâniche
}

Published online: 21 February 2012

(C) The Brazilian Computer Society 2012

This Special Issue of the Journal of the Brazilian Computer Society features extended versions of five papers selected from The fifth Latin American Dependable Computing (LADC'2011) symposium, which took place in São José dos Campos, SP, on April 25-29, 2011.

LADC is the major Latin American event that provides a forum for researchers and practitioners from all over the world to present and discuss their latest research results and developments on the dependability and security of computer systems and networks. The symposium scope spans system, software and hardware dependability, as well as security research and technological challenges, encompassing fundamental theoretical approaches and practical experimental studies.

LADC'2011 received a total of 46 submissions from 12 countries. The entire process was handled electronically. A total of 159 reviews were entered, each paper received at least three reviews. The review process included a rebuttal period during which the authors had the opportunity to clarify doubts and other issues raised in the reviews of their papers. After the rebuttal period, the TPC members started the discussion phase, which resulted in the selection of the 18 papers that are included in the technical program (16 Regular Papers, and 2 Practical Experience Re-

\footnotetext{
L.E. Buzato

IC-UNICAMP, Campinas, Brazil

e-mail: buzato@ic.unicamp.br

E.P. Duarte Jr.

Federal University of Paraná, Curitiba, Brazil

e-mail: elias@inf.ufpr.br

M. Kaâniche ( $₫)$

LAAS-CNRS, University of Toulouse, Toulouse, France

e-mail: Mohamed.Kaaniche@laas.fr
}

ports). The selected papers cover various areas of dependable computing, including fault tolerance and diagnosis in distributed and mobile systems, security, verification, experimental analysis and evaluation. From those 18 papers five were invited to be extended for publication in this JBCS Special Issue.

The first paper, "Byzantine Fault-Tolerant Deferred Update Replication" by Fernando Pedone and Nicolas Schiper, considers the use of deferred update replication in settings with byzantine failures. The paper makes three contributions. First, it shows that making deferred update replication tolerate byzantine failures is quite simple. Second, the paper presents a byzantine-tolerant mechanism to execute read-only transactions at a single server. Third, we consider byzantine client attacks against deferred update replication and discuss effective countermeasures against these attacks.

The second paper, "Energy-Aware Test Connection Assignment for the Self-Diagnosis of a Wireless Sensor Network" by Andrea Weber, Alexander R. Kutzke, and Stefano Chessa, proposes a system-level diagnosis strategy for longterm monitoring of Wireless Sensor Networks, in order to detect node malfunctions quickly and with an energy efficient way. An algorithm for the assignment of tests among the sensors of a WSN that ensures the desired system diagnosability and that is aware of energy consumption.

The third paper, "Timing Analysis of Leader-based and Decentralized Byzantine Consensus Algorithms" by Fatemeh Borran, Martin Hutle, and André Schiper. This paper deals with the problem of how to compare the time performance of byzantine consensus algorithms in a partially synchronous system with strong validity. The authors compare analytically, in a round-based model, a leader-based variant of byzatine consensus with its decentralized variant. The paper shows that, in most cases, the decentralized variant of the algorithm has a better worst-case execution time. 
The fourth paper, "Exception Handling System for Service Component Architectures" by Fernando Castor, Douglas Siqueira Leite and Cecília Mary F. Rubira, presents the design and implementation of an exception-handling model that target Service Component Architectures (SCA). The proposed model supports coordinated exception handling and allows the creation, in a flexible way, of faulttolerant asynchronous service compositions in SCA implementations. This model is designed to be easily instantiated to new and existing applications using aspect-oriented programming.

The fifth paper, "On the Reliability and Availability of Replicated and Rejuvenating Systems Under Stealth Attacks and Intrusions", by Luís Teixeira d'Aguiar Norton Brandão and Alysson Neves Bessani, applies probabilistic reliability and availability modeling techniques in the context of intrusion tolerance. The paper compares several state-of-theart intrusion tolerance strategies using replication and rejuvenation, taking into account different metrics and parameters including reliability, availability, mission time, expected time to node intrusion, etc., and considering different attack strategies. In particular, the models highlight the significant impact of mission time and rejuvenation and identify scenarios where intrusion tolerance strategies can lead to reliability or availability degradation when compared to a non replicated system.

Special thanks go to JBCS Editor-In-Chief, Maria Cristina de Oliveira, Ana Maria Ambrosio, the LADC'2011 General Chair, and the Organizing, Steering and Program Committees for their outstanding support to the conference.

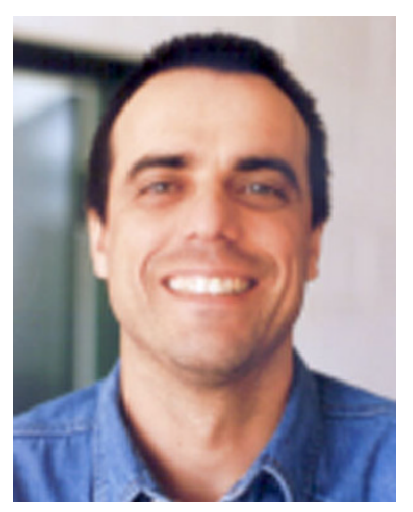

Luiz Eduardo Buzato

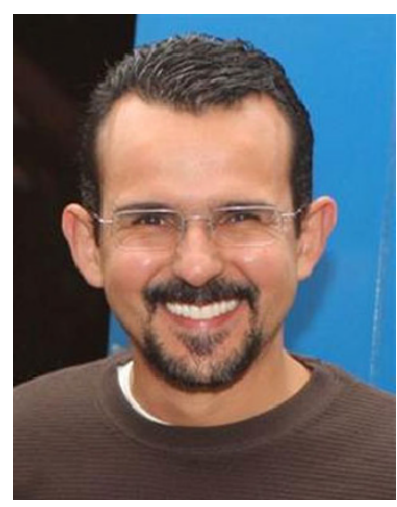

Elias P. Duarte Jr.

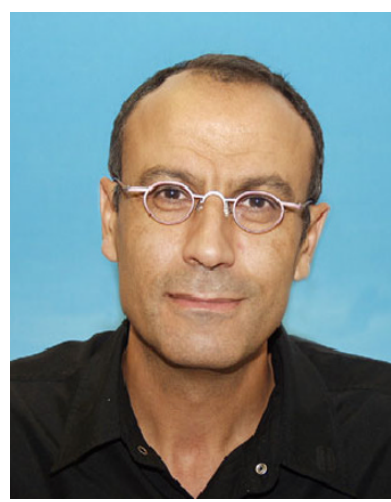

Mohamed Kaâniche 